OPEN ACCESS

Edited by:

John J. Miles,

James Cook University, Australia

Reviewed by:

Kenichi Hanada,

National Institutes of Health (NIH),

United States

Jamie Berta Spangler Johns Hopkins University,

United States

*Correspondence:

Gideon Gross

gidi@migal.org.il

Specialty section:

This article was submitted to

T Cell Biology,

a section of the journal

Frontiers in Immunology

Received: 21 September 2021

Accepted: 21 October 2021

Published: 04 November 2021

Citation:

Savanur MA, Weinstein-Marom H and Gross G (2021) Implementing

Logic Gates for Safer

Immunotherapy of Cancer.

Front. Immunol. 12:780399. doi: 10.3389/fimmu.2021.780399

\section{Implementing Logic Gates for Safer Immunotherapy of Cancer}

\author{
Mohammed Azharuddin Savanur ${ }^{1,2}$, Hadas Weinstein-Marom ${ }^{1,2}$ and Gideon Gross ${ }^{1,2 *}$ \\ ${ }^{1}$ Laboratory of Immunology, MIGAL - Galilee Research Institute, Kiryat Shmona, Israel, 2 Department of Biotechnology, \\ Tel-Hai College, Upper Galilee, Israel
}

Targeting solid tumors with absolute precision is a long-standing challenge in cancer immunotherapy. The identification of antigens, which are expressed by a large fraction of tumors of a given type and, preferably, across various types, but not by normal cells, holds the key to developing safe, off-the-shelf immunotherapies. Although the quest for widely shared, strictly tumor-specific antigens has been the focus of tremendous effort, only few such candidates have been implicated. Almost all antigens that are currently explored as targets for chimeric antigen receptor (CAR) or T cell receptor (TCR)-T cell therapy are also expressed by healthy cells and the risk of on-target off-tumor toxicity has remained a major concern. Recent studies suggest that this risk could be obviated by targeting instead combinations of two or more antigens, which are co-expressed by tumor but not normal cells and, as such, are tumor-specific. Moreover, the expression of a shared tumor antigen along with the lack of a second antigen that is expressed by normal tissues can also be exploited for precise recognition. Additional cues, antigenic or non-antigenic ones, which characterize the tumor microenvironment, could be harnessed to further increase precision. This review focuses on attempts to define the targetable signatures of tumors and assesses different strategies employing advanced synthetic biology for translating such information into safer modes of immunotherapy, implementing the principles of Boolean logic gates.

Keywords: Boolean logic gates, adoptive cell therapy, chimeric antigen receptors, on-target off-tumor toxicity, synthetic biology

\section{INTRODUCTION}

In adoptive cell therapy (ACT) of cancer, great effort is made to develop off-the-shelf genes, designed for redirecting autologous $\mathrm{T}$ or NK cells to selectively eradicate tumor cells while avoiding on-target off-tumor attack (1-3). Ready-to-use, donor-derived T/NK cells genetically reprogrammed to recognize a given type of cancer will vastly accelerate treatment and assure quality and quantity of the cell product (4). Chimeric antigen receptors [CARs, originally developed by $\mathrm{G}$. $\mathrm{G}$. and colleagues $(5,6)$ ] redirect $\mathrm{T}$, and other immune killer cells to recognize antigens in an MHC-independent manner and are thus ideally suited for this purpose. However, the vast majority of CAR antigens expressed on solid tumors or cellular components of the tumor microenvironment (TME) that are presently investigated are expressed by vital nontumor tissues (7-11) and their targeting poses a severe safety concern. This critical downside has prompted the search for 
combinations of antigens (present or absent) as well as nonantigenic cues, which could define new targetable tumor-specific signatures. To achieve this goal two major challenges are concomitantly addressed: 1) Identification of combinations, which are shared by the vast majority, if not all tumor cells in a large fraction of patients and can unequivocally discriminate between tumor and nontumor tissues. 2) The development of molecular devices, which can sense and integrate these complex inputs to produce the desired biological output, implementing the basic principles of Boolean logic gates. Two types of logic gates that are particularly relevant to the safety challenge are the logic AND gate, which would only produce an output in the presence of all its designated inputs and the NOT gate, which would require the presence of at least one input and the absence of another, to act. Here we examine different classes of such cues as potential components of tumor-specific signatures and review various strategies used to exploit the AND and NOT gates for their immunotargeting. Other powerful genetic approaches implementing the OR logic gate (12-15), TRUCKs (16-18) or switch receptors (19-22) address additional critical challenges in ACT and are not dealt with in this review.

\section{POTENTIAL LOGIC GATE INPUTS}

\section{Antigenic Cues}

While distinct input and output modules of logic gated ACT are defined by their biological thresholds, each intact circuit should eventually comply with clinical tolerability. This aspect is particularly important while weighing the actual expression of a given antigen by tumor vs. normal cells, as the outcome of antigen binding critically depends not only on its expression level, but also on the overall avidity of interaction, the fidelity of the signaling cascade and the response mechanism. With this in mind, attempts are made to identify combinations of surface antigens that are expressed by all cells in a given tumor and by most tumors of a given type and, desirably, by different tumor types, but not by any vital tissue. As the number of input signals grows, precision is expected to increase, but at the expense of the fraction of tumors complying with all inputs and of applicability, as additional modules would inevitably require increasingly improved gene transfer vectors.

Acute myeloid leukemia (AML) is a heterogeneous class of diseases characterized by particularly high genetic instability that gives rise to multiple immunophenotypes in a single patient, rendering AML notoriously refractory to conventional immunotherapies (23). In attempt to circumvent this heterogeneity, Perna et al. (24) performed meticulous proteomics and transcriptomics analysis of large surfaceome datasets from malignant and normal tissues, aiming at identifying antigen pairs which could serve as targets for combinatorial CAR therapy. While a number of antigens have been previously reported as potential AML CAR targets, none met the criteria defined in this study for an optimal single target. Following a stringent refining process, four antigen pairs: ADGRE2+CD33, CCR1+CLEC12A, CD70+CD33, and
LILRB2+CLEC12A, yielded nearly optimal results, for all of which the dual targeting score significantly exceeded that of either antigen alone.

More recently, Dannenfelser et al. carried out a comprehensive in-silico screen of $>2.5$ million dual and 60 million triple antigen combinations across 33 tumor types and 34 normal tissues, assessing their ability to discriminate tumors from normal tissues via either AND or NOT gates (25). For prioritizing candidate antigen combinations the authors created a numerical ruler, integrating precision and recall (fraction of targetable tumor samples), with which they compared single antigens to double and triple gates. For every cancer type assessed they identified at least 25 high-score antigen pairs, which substantially outperform single 'clinical' antigens currently explored in CAR therapy of the same cancer. Furthermore, for several tumor types, triple antigen combinations show near ideal precision, yet, with an anticipated decline in recall. Although this study is based on RNA-seq databases rather than actual surface protein expression, it underscores the discriminatory power of combinatorial antigen recognition, paving the way for clinical evaluation of numerous CAR therapies targeting new antigen combinations in virtually all cancer types.

Another class of candidate antigens for combinatorial recognition are expressed by tumor-supporting cells at the TME, including cancer-associated fibroblasts, tumor-associated macrophages, myeloid-derived suppressor cells, regulatory $\mathrm{T}$ cells and additional immune cells (9-11), as well as tumor endothelial cells (TECs) (26-29). Antigens that are preferentially expressed by these cells (such as VEGFRs, PSMA, ALCAM, CD13, CLEC14A, RGS5, TEMs, fibronectin EIIIB splice variant, Endothelin $B$ receptor, $\alpha_{v} \beta_{3}$ integrin and others) are particularly attractive, as, unlike tumor antigens, they are not subjected to genetic and epigenetic instability. Among these, TECs draw special attention, as they are highly accessible to the therapeutic cells and are less affected by immunesuppressive conditions dominating the TME. While none of these antigens is utterly specific, collectively they provide an additional layer of potential targets for gated therapy.

Loss of heterozygosity $(\mathrm{LOH})$ characterizes the majority of human cancers. It is manifested in multiple losses of full chromosomes, entire chromosomal arms or sub-chromosomal regions and is often associated with the loss of a normal copy of a tumor suppressor gene (30-33). Many LOH events already occur prior to malignant transformation creating a loss signature that is shared by premalignant cells and all descendant tumor cells in a given patient (34). An inevitable outcome of $\mathrm{LOH}$ is the concomitant loss of all other genes residing on the deleted chromosomal material, and these naturally include heterozygous alleles of protein-coding genes. This early $\mathrm{LOH}$ driven antigenic landscape is consequently irreversible and is not affected by tumor heterogeneity. LOH-based discrimination of tumor from normal cells can be achieved by NOT gates based on either CARs that are directed at an allelic variant encoding an extracellular epitope, which can be distinguished from the homologous one or TCRs, recognizing a linear peptide that is 
presented on normal but not tumor cells by one of the patient's HLA-I products. A special class of LOH events involve the HLA gene locus on chromosome 6q21 and are apparently exploited by tumors as an escape mechanism from CD8 T cells (35-37). It now emerges that this mechanism also allows tumor cells to avoid recognition by adoptively transferred $\mathrm{T}$ cells, as reported, for example, in TIL therapy of a patient with metastatic colorectal cancer, targeting a peptide harboring the KRAS G12D mutation presented by HLA-C ${ }^{\star} 08: 02$ (38). LOH is source for a universe of antigenic combinations that can be targeted by NOT modules and pioneering attempts to explore this new route to cancer immunotherapy have recently been published $(39,40)$.

\section{Non-Antigenic Cues at the TME}

Hypoxia is a prominent feature of the TME, resulting from rapidly dividing cancer cells and aberrant vascularization (41). Tumor cell adaptation to hypoxic conditions is governed by hypoxia-inducible factors (HIFs) via the hypoxia pathway (42). Under normoxic conditions, prolyl-hydroxylases (PHDs) hydroxylates conserved prolines of HIF-1 $\alpha$, leading to ubiquitination and proteasomal degradation. However, during hypoxic conditions, the PHDs are inactive, allowing HIF-1 $\alpha$ accumulation, translocation to the nucleus, dimerization with HIF-1 $\beta$ and formation of a transcriptional complex. The HIF complex binds to the promoter region of hypoxia-responsive elements (HRE) and triggers transcription, which regulates several biological functions of tumor cells (43). These two hypoxia-induced mechanisms, acting independently at the transcription and post-translational levels, can be exploited for confining CAR activity to hypoxic tissues.

Pro-inflammatory cytokines secreted by a variety of cells at the TME induce cellular responses that are associated with tumor initiation, progression, tissue invasion, metastasis and evasion from an immune response. In parallel, potent immunosuppressive mediators that are overproduced by tumor-supportive immune cells act in many solid tumors to counteract the antitumor response. Consequently, the TME is often characterized by elevated levels of cytokines, including TNF-?, IFN- $\gamma$, IL-6, IL-8, IL-17, IL-21, TGF-? and IL-10 (44-46), which can serve as TME biomarkers and guide AND gated therapies exploiting cytokineresponsive receptors or transcriptional control elements.

Continuous interactions between tumor and nontumor cells at the TME result in the upregulation of a battery of pericellular proteases, which play a critical role in tumor angiogenesis, invasion and metastasis (47-49). These include various matrix metalloproteinases, cathepsins, elastase, granzyme B, tissue and urokinase plasminogen activators and others, creating a proteaserich niche that is distinguishable from most other normal tissues. This unique characteristic of the TME paves the way for the design of antitumor antibodies or CARs whose recognition moieties are masked by a protease-cleavable peptide, which is liberated at the tumor site, creating a bona-fide logic AND gate.

Tumor-specific as well as tissue- or state-specific promoters can be harnessed to confine the expression of therapeutic genes of interest (GOIs) to designated target cells in-vivo and, as such, offer another class of cues for gated cancer therapy. However, as with tumor antigens, no single promoter appears to be solely restricted to a given type of tumor. Nonetheless, dividing the specificity task between two promoters, both simultaneously active only in the target cells, could confer the desired precision of action (50-52). In addition, synthetic promoters comprising distinct transcription factor (TF)-binding elements can assure that transcription of a therapeutic gene takes place only in cells expressing all corresponding TFs $(53,54)$.

\section{SELECTED STRATEGIES}

\section{AND Logic Gates Combinatorial Antigen Recognition}

The 'split' recognition AND gate (Figure 1) comprises an affinityreduced CAR specific to one antigen that harbors an activation domain (typically $\mathrm{CD} 3 \zeta$ ) only, co-expressed with a chimeric costimulatory receptor (CCR) that is specific to another antigen, which only possesses a costimulatory element (often derived from CD28 and/or 4-1BB), but no activation domain. If properly calibrated, split antigen recognition would allow full $\mathrm{T}$ cell activation only in the presence of both antigens, while normal cells expressing only one would be protected. This principle has been demonstrated in several preclinical studies [e.g (55-58)].

SUPRA CARs (Figure 1) logically respond to multiple antigens without the need of re-engineering T cells specifically for each target antigen (59). The universal zipCAR module possesses a conventional CAR signaling moiety connected to an extracellular leucine zipper. The zipFv antigen-recognition module harbors a scFv fused to a second leucine zipper, which is capable of interacting with the zipCAR. The engineering of multiple zippers with varying binding properties enables multiplexed control over $\mathrm{T}$ cell responses, including a split-CAR configuration.

The RevCAR platform (Figure 1) (60) consists of compact CARs incorporating peptide tags instead of full-length $\mathrm{scFv}$, and bispecific molecules coupling an anti-tag $\mathrm{scFv}$ to an antitumor scFv. Using two split anti-tag CARs and the corresponding bispecific engagers the system operates as an AND gate.

Reduced affinity CARs are also the central components of the AvidCAR AND gate strategy (Figure 1) (61). It exploits monomeric, reduced affinity CARs specific to one antigen, designed to homodimerize by the second antigen through a second scFv incorporated into the same ectodomain, thereby gaining sufficient avidity for $\mathrm{T}$ cell activation.

The 'Co-LOCKR' gate (Figure 1) (62), is based on competition, rather than complementation. This system employs a universal CAR recognizing a unique 'recruitment peptide' which resides on a 'Latch' domain engrafted onto a protein termed the 'Cage' that is capable of binding antigen $\mathrm{A}$. In both the unbound and the bound states of the Cage the recruitment peptide is sequestered by the Cage-bound Latch and cannot bind its receptor. The second soluble protein component of this system is the 'Key'. It recognizes antigen $\mathrm{B}$ and contains a unique domain, which can compete out the folded sequestering domain of the Cage and 


\section{AND gates}

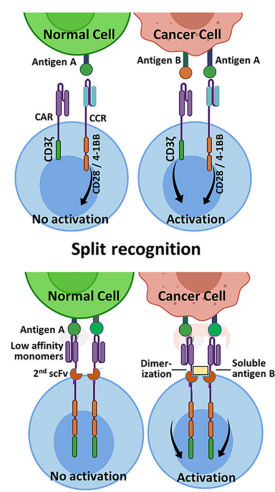

AvidCAR

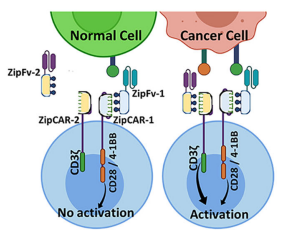

SUPRA CAR

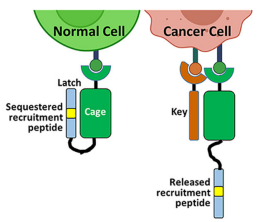

Co-LOCKR

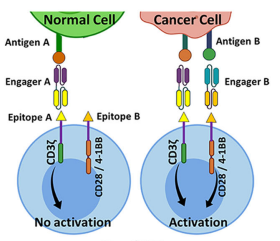

RevCAR

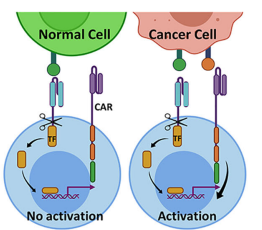

SynNotch
NOT gates
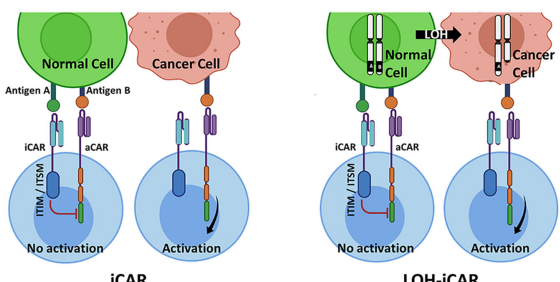

LOH-iCAR

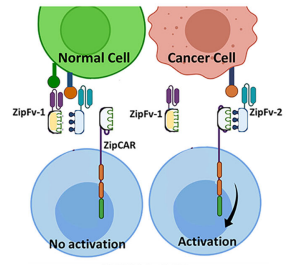

SUPRA CAR
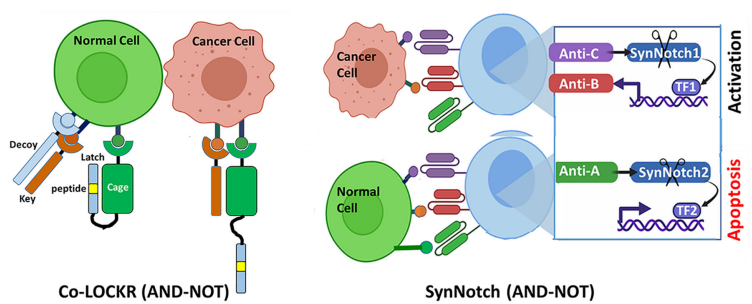

FIGURE 1 | Schematic representation of selected Boolean logic AND and NOT gate strategies for integrating two or three antigenic cues, designed to enhance precision of CAR-T cell therapy of cancer.

AND gates. The split recognition strategy involves one receptor with an activation domain and a second receptor with a co-stimulatory domain, each specific to a different antigen. Full T cell activation is only achieved upon simultaneous engagement of the two receptors with their respective antigens. Each SUPRA CAR system utilizes a universal CAR-like receptor (zipCAR) carrying a leucine zipper, which can bind a corresponding leucine zipper incorporated onto a soluble antigen-specific zipFv protein, enabling multiplexed control of T cell responses. Shown here are zipCAR-1, equipped with a leucine zipper specific to that of zipFv-1, which recognizes antigen A that is shared by the tumor and normal cell, and zipCAR-2, specific to zipFv-2 and to antigen B that is expressed by the tumor cell but not by the same normal cell. RevCARs are economical CAR constructs, harboring peptide epitopes instead of scFvs. The RevCAR platform utilizes bi-specific engagers, each comprising two scFvs: one specific to a distinct peptide epitope and the other recognizing a tumor-associated antigen. Shown here are a T cell co-expressing two different RevCARs, each harboring a different peptide epitope and either T cell activation or costimulatory domain, and two engagers, each directed at a given peptide epitope and a tumor antigen. The T cell can only be activated when the two engagers are simultaneously bound to their tumor antigens. AvidCAR is an avidity-controlled CAR platform, designed to homodimerize upon dual antigen recognition conferring sufficient avidity for $T$ cell activation. The Co-LOCKR gate utilizes a universal CAR specific to a recruitment peptide, which is placed in a 'Latch' sequence. The latch itself is linked to a soluble 'Cage' protein that is directed at first tumor antigen. The recruitment peptide is normally sequestered by the Cage and is only exposed to engagement by the CAR when a competing 'Key' protein binds a second antigen present on the same tumor cell. The synNotch AND gate comprises a constitutively expressed module which releases a synthetic TF only upon binding of antigen A. Upon translocation to the T cell nucleus, this TF drives the expression of a conventional CAR specific to antigen B.

NOT gates. The iCAR and the LOH-iCAR typically utilize an inhibitory element derived from a T/NK cell inhibitory receptor. Binding of the iCAR to the inhibitory antigen would prevent T cell activation that could otherwise take place following engagement of the aCAR with the activating antigen. The SUPRA CAR NOT module operates through competition: when both zipFv-1 and zipFv-2 proteins bind their respective antigens A and B on normal cells, their complementary leucine zippers are tightly engaged, precluding activation of the zipCAR. T cell activation can only take place upon encounter with tumor cells, in the absence of competition by nonbound zipFv-1. The Co-LOCKR NOT circuit implements a combination of AND and NOT gates and functions through competition, employing a third protein (in addition to the Cage and Key proteins) that binds specifically to a third antigen present on normal but not tumor cells. This protein possesses a Decoy element, which binds Key with high affinity. When all three proteins are engaged with their target epitopes on the surface of a normal cell, Key is prevented from binding to Cage so that release of the recruitment peptide does not take place. The synNotch circuit shown here utilizes a suicide module, which is only activated upon encounter with an inhibitory antigen expressed by the normal cell to be protected. 
liberate the recruitment peptide that is now accessible to the CAR. This productive interaction can only take place if antigens $\mathrm{A}$ and $\mathrm{B}$ are present in close proximity on the same target cell and does not occur in solution.

\section{AND Gates Utilizing Synthetic Promoters and Non-Antigenic Cues}

The synthetic Notch (synNotch)-based AND gate, (Figure 1 (63-66), and see (67) for review), harnesses regulated intramembrane proteolysis (68), a unique signaling pathway that utilizes the dual cleavage of a group of cell surface receptors, including Notch, by membrane-associated proteases following ligand binding. This process releases the intracellular domain (ICD), which can then translocate to the cell nucleus and function as a TF. The basic synNotch module encodes an $\mathrm{N}$ terminal $\mathrm{scFv}$ targeting antigen $\mathrm{A}$, fused to a minimal Notch sequence including the cleavable transmembrane domain and a microbial-derived ICD, which can operate as a TF that controls gene expression from a synthetic promoter. The second module encodes a conventional CAR targeting antigen $\mathrm{B}$ and is placed under the control of this synthetic promoter, so that the entire circuit only operates in the presence of both antigens. By expressing a synNotch receptor with low-affinity for a given antigen, which controls the expression of a high affinity CAR against the same antigen, Hernandez-Lopez et al. recently engineered $\mathrm{T}$ cells to discriminate with high sensitivity between tumor cells overexpressing HER2 and normal cells displaying low HER2 density (69).

In 2017, Nissim et al. presented a revolutionary AND gate comprising a two-module mRNA circuit, which they used for the in-vivo delivery of a multi-component immunostimulatory cassette to be exclusively expressed in cancer cells (51). The expression of the GOI is governed by the coordinated activity of two separate modules, each driven by one of two synthetic promoters, which are simultaneously active in cancer cells only. While operating here as a multi-functional immune modulator, this approach offers numerous gated applications in ACT.

Several strategies have exploited hypoxia-induced pathways for designing self-decision-making CAR-T cells. One such approach utilizes CARs that incorporate an oxygen-sensing subdomain (HIF-1 $\alpha$ ) into the CAR scaffold and become active only under hypoxic conditions (70). Another strategy restricts CAR expression to the TME by introducing HRE regions into the CAR promoter, thus coupling transcription to hypoxia (71).

A synthetic promoter [dubbed CARTIV (54)], places a CAR gene under the control of inflammation and hypoxia-induced signals characterizing the TME. To obtain a proof of concept, the authors incorporated into these promoters DNA elements responsive to IFN $\gamma, \mathrm{TNF} \alpha$ and hypoxia and showed an additive effect on CAR expression and function in primary human $\mathrm{T}$ cells in the presence of the three stimuli.

A unique AND gate integrating inputs from the TEC-associated promoter of the pre-proendothelin-1 gene and the local presence of TNF $\alpha$, directs the in-vivo expression of a suicide Fas-TNFR1 receptor selectively to TECs, exerts an antiangiogenic effect and shows promising efficacy in clinical studies $(72,73)$.

'Probodies' are soluble antitumor mAb-based prodrugs, which function as two-module AND gates (74). While the antigenic cue is targeted by the probody antigen-binding site, it is masked by a cleavable peptide, designed for preferential removal at the protease-rich TME. The therapeutic potential of probodies was initially demonstrated in a human xenograft model for non-small cell lung cancer, using the anti-EGFR antibody cetuximab (75) and was later applied to antitumor CARs, using essentially the same molecular and experimental design (76).

\section{NOT Logic Gates}

NOT gates are designed to protect normal cells that express a selected tumor antigen targeted by an activating CAR (aCAR) or TCR), as well as a second antigen (the 'protective' antigen) that is NOT expressed by the tumor. An effective NOT device would counteract $\mathrm{T}$ cell activation triggered by the tumor antigen when simultaneously engaging the protective antigen on the same normal cell. Creating a safe NOT gate entails the incorporation of an inhibitory module specific to the second antigen, which can operate through either strong inhibitory signaling or potent competition with the activating module while guaranteeing continuous dominance over the latter. It is mandatory that the effect of the NOT module on the therapeutic T cell is transient and fully reversible, as repeated inhibitory signaling can anergize these cells and abolish their therapeutic efficacy.

The implementation of the inhibitory approach for producing a NOT gate was pioneered by Fedorov at al (77)., who recruited the signaling domains of the T cell inhibitory receptors CTLA-4 and PD-1 to create inhibitory CARs (iCARs) (Figure 1). Since then a number of studies exploring NOT gates have examined iCARs incorporating inhibitory signaling elements that are derived from $\mathrm{T}$ or $\mathrm{NK}$ cell-associated inhibitory receptors, including PD-1 (40, 78), BTLA (79) and LIR1 (39).

Employing competitive inhibition, the SUPRA CAR technology offers a 'Cell Selector' application (Figure 1) (59). Here, the presence of a protective antigen on normal cells recruits a designated zipFv harboring a zipper, which binds avidly to the activating zipFv zipper, blocking its binding to the zipCAR, thereby preventing activation. Another competition-based NOT module is offered by the Co-LOCKR system (Figure 1), allowing the simultaneous recognition of antigen A AND antigen B but NOT antigen C, creating an AND-NOT gate (62). This scenario is addressed through a third soluble component specific to antigen C, which carries a 'Decoy' segment. Upon antigen binding on the cell to be protected, the Decoy binds tightly to the adjacent 'Key' and prevents binding of the latter to the 'Cage', so that release of the recruitment peptide and subsequent $\mathrm{T}$ cell activation cannot take place. Another antigen-driven AND-NOT circuit was recently reported (Figure 1), utilizing a synNotch receptor, which governs the expression of the pro-apoptotic factor tBID (truncated BID, a member of the BH3-domain-only subgroup of BCL-2 family) upon antigen binding as a novel, gated suicide mechanism (66). 


\section{CONCLUSIONS}

Exploiting combinations of shared antigenic and non-antigenic features characterizing all types of cancer by logic-gated technologies can dramatically increase the fidelity of tumor targeting and alleviate safety concerns. While advancing exciting gates that combine universal CARs with soluble proteins (CoLOCKR, SUPRA CARs, RevCARs) still requires extensive calibration and optimization, others, such as split recognition or iCARs seem closer to clinical evaluation. New and powerful bioinformatic algorithms, rapidly growing datasets of tumors vs. normal cells, the development of sophisticated synthetic biology tools, along with progress made in our understanding of the

\section{REFERENCES}

1. Yang JC, Rosenberg SA. Adoptive T-Cell Therapy for Cancer. Adv Immunol (2016) 130:279-94. doi: 10.1016/bs.ai.2015.12.006

2. Abken H. Driving CARs on the Highway to Solid Cancer: Some Considerations on the Adoptive Therapy With CAR T Cells. Hum Gene Ther (2017) 28:1047-60. doi: 10.1089/hum.2017.115

3. Leko V, Rosenberg SA. Identifying and Targeting Human Tumor Antigens for T Cell-Based Immunotherapy of Solid Tumors. Cancer Cell (2020) 38:454-72. doi: 10.1016/j.ccell.2020.07.013

4. Caldwell KJ, Gottschalk S, Talleur AC. Allogeneic CAR Cell Therapy-More Than a Pipe Dream. Front Immunol (2020) 11:618427. doi: 10.3389/ fimmu.2020.618427

5. Gross G, Waks T, Eshhar Z. Expression of Immunoglobulin-T-Cell Receptor Chimeric Molecules as Functional Receptors With Antibody-Type Specificity. Proc Natl Acad Sci USA (1989) 86:10024-8. doi: 10.1073/pnas.86.24.10024

6. Eshhar Z, Waks T, Gross G, Schindler DG. Specific Activation and Targeting of Cytotoxic Lymphocytes Through Chimeric Single Chains Consisting of Antibody-Binding Domains and the Gamma or Zeta Subunits of the Immunoglobulin and T-Cell Receptors. Proc Natl Acad Sci USA (1993) 90:720-4. doi: 10.1073/pnas.90.2.720

7. Gross G, Eshhar Z. Therapeutic Potential of T Cell Chimeric Antigen Receptors (CARs) in Cancer Treatment: Counteracting Off-Tumor Toxicities for Safe CAR T Cell Therapy. Annu Rev Pharmacol Toxicol (2016) 56:59-83. doi: 10.1146/annurev-pharmtox-010814-124844

8. Caruso HG, Heimberger AB, Cooper LJN. Steering CAR T Cells to Distinguish Friend From Foe. Oncoimmunology (2019) 8:e1271857. doi: 10.1080/2162402X.2016.1271857

9. Van der Jeught K, Bialkowski L, Daszkiewicz L, Broos K, Goyvaerts C, Renmans D, et al. Targeting the Tumor Microenvironment to Enhance Antitumor Immune Responses. Oncotarget (2015) 6:1359-81. doi: 10.18632/oncotarget.3204

10. Pitt JM, Marabelle A, Eggermont A, Soria J-C, Kroemer G, Zitvogel L. Targeting the Tumor Microenvironment: Removing Obstruction to Anticancer Immune Responses and Immunotherapy. Ann Oncol Off J Eur Soc Med Oncol (2016) 27:1482-92. doi: 10.1093/annonc/mdw168

11. Rodriguez-Garcia A, Palazon A, Noguera-Ortega E, Powell DJ, Guedan S. CART Cells Hit the Tumor Microenvironment: Strategies to Overcome Tumor Escape. Front Immunol (2020) 11:1109. doi: 10.3389/fimmu.2020.01109

12. Grada Z, Hegde M, Byrd T, Shaffer DR, Ghazi A, Brawley VS, et al. TanCAR: A Novel Bispecific Chimeric Antigen Receptor for Cancer Immunotherapy. Mol Ther Nucleic Acids (2013) 2:e105. doi: 10.1038/mtna.2013.32

13. Hegde M, Mukherjee M, Grada Z, Pignata A, Landi D, Navai SA, et al. Tandem CAR T Cells Targeting HER2 and IL13R 22 Mitigate Tumor Antigen Escape. J Clin Invest (2016) 126:3036-52. doi: 10.1172/JCI83416

14. Zah E, Lin M-Y, Silva-Benedict A, Jensen MC, Chen YY. T Cells Expressing CD19/CD20 Bispecific Chimeric Antigen Receptors Prevent Antigen Escape by Malignant B Cells. Cancer Immunol Res (2016) 4:498-508. doi: 10.1158/ 2326-6066.CIR-15-0231 intricate immune recognition and signaling networks, all pave the way for the creation of increasingly powerful logic gates for improved precision of cancer immunotherapy.

\section{AUTHOR CONTRIBUTIONS}

MS and GG wrote the initial text and prepared the accompanying figure. HW-M carefully reviewed the text, made constructive comments, and helped shape the final version of the manuscript. All authors contributed to the article and approved the submitted version.
15. Martyniszyn A, Krahl A-C, André MC, Hombach AA, Abken H. CD20-CD19 Bispecific CAR T Cells for the Treatment of B-Cell Malignancies. Hum Gene Ther (2017) 28:1147-57. doi: 10.1089/hum.2017.126

16. Chmielewski M, Kopecky C, Hombach AA, Abken H. IL-12 Release by Engineered T Cells Expressing Chimeric Antigen Receptors Can Effectively Muster an Antigen-Independent Macrophage Response on Tumor Cells That Have Shut Down Tumor Antigen Expression. Cancer Res (2011) 71:5697-706. doi: 10.1158/0008-5472.CAN-11-0103

17. Zhang L, Kerkar SP, Yu Z, Zheng Z, Yang S, Restifo NP, et al. Improving Adoptive $\mathrm{T}$ Cell Therapy by Targeting and Controlling IL-12 Expression to the Tumor Environment. Mol Ther (2011) 19:751-9. doi: 10.1038/ mt.2010.313 10.1038/mt.2010.313.

18. Chmielewski M, Abken H. TRUCKs: The Fourth Generation of CARs. Expert Opin Biol Ther (2015) 15:1145-54. doi: 10.1517/14712598.2015.1046430

19. Prosser ME, Brown CE, Shami AF, Forman SJ, Jensen MC. Tumor PD-L1 CoStimulates Primary Human CD8(+) Cytotoxic T Cells Modified to Express a PD1:CD28 Chimeric Receptor. Mol Immunol (2012) 51:263-72. doi: 10.1016/ j.molimm.2012.03.023

20. Ankri C, Shamalov K, Horovitz-Fried M, Mauer S, Cohen CJ. Human T Cells Engineered to Express a Programmed Death 1/28 Costimulatory Retargeting Molecule Display Enhanced Antitumor Activity. J Immunol (2013) 191:4121-9. doi: 10.4049/jimmunol.1203085

21. Tay JC, Zha S, Wang S. Chimeric Switch Receptor: Switching for Improved Adoptive T-Cell Therapy Against Cancers. Immunotherapy (2017) 9:1339-49. doi: 10.2217/imt-2017-0103

22. Guo J, Kent A, Davila E. Chimeric Non-Antigen Receptors in T Cell-Based Cancer Therapy. J Immunother Cancer (2021) 9. doi: 10.1136/jitc-2021-002628

23. Romer-Seibert JS, Meyer SE. Genetic Heterogeneity and Clonal Evolution in Acute Myeloid Leukemia. Curr Opin Hematol (2021) 28:64-70. doi: 10.1097/ MOH.0000000000000626

24. Perna F, Berman SH, Soni RK, Mansilla-Soto J, Eyquem J, Hamieh M, et al. Integrating Proteomics and Transcriptomics for Systematic Combinatorial Chimeric Antigen Receptor Therapy of AML. Cancer Cell (2017) 32:506-19. doi: 10.1016/j.ccell.2017.09.004

25. Dannenfelser R, Allen GM, VanderSluis B, Koegel AK, Levinson S, Stark SR, et al. Discriminatory Power of Combinatorial Antigen Recognition in Cancer T Cell Therapies. Cell Syst (2020) 11:215-228.e5. doi: 10.1016/j.cels.2020.08.002

26. Zhao Y, Yu X, Li J. Manipulation of Immune-Vascular Crosstalk: New Strategies Towards Cancer Treatment. Acta Pharm Sin B (2020) 10:201836. doi: 10.1016/j.apsb.2020.09.014

27. Akbari P, Huijbers EJM, Themeli M, Griffioen AW, van Beijnum JR. The Tumor Vasculature an Attractive CAR T Cell Target in Solid Tumors. Angiogenesis (2019) 22:473-5. doi: 10.1007/s10456-019-09687-9

28. Liu G, Rui W, Zhao X, Lin X. Enhancing CAR-T Cell Efficacy in Solid Tumors by Targeting the Tumor Microenvironment. Cell Mol Immunol (2021) 18:1085-95. doi: 10.1038/s41423-021-00655-2

29. Fuca G, Reppel L, Landoni E, Savoldo B, Dotti G. Enhancing Chimeric Antigen Receptor T-Cell Efficacy in Solid Tumors. Clin Cancer Res (2020) 26:2444-51. doi: 10.1158/1078-0432.CCR-19-1835 
30. Devilee P, Cleton-Jansen A-M, Cornelisse CJ. Ever Since Knudson. Trends Genet (2001) 17:569-73. doi: 10.1016/S0168-9525(01)02416-7

31. Lengauer C, Kinzler KW, Vogelstein B. Genetic Instabilities in Human Cancers. Nature (1998) 396:643-9. doi: 10.1038/25292

32. Beroukhim R, Mermel CH, Porter D, Wei G, Raychaudhuri S, Donovan J, et al. The Landscape of Somatic Copy-Number Alteration Across Human Cancers. Nature (2010) 463:899-905. doi: 10.1038/nature08822

33. Knouse KA, Davoli T, Elledge SJ, Amon A. Aneuploidy in Cancer: Seq-Ing Answers to Old Questions. Annu Rev Cancer Biol (2017) 1:335-54. doi: 10.1146/annurev-cancerbio-042616-072231

34. Gerstung M, Jolly C, Leshchiner I, Dentro SC, Gonzalez S, Rosebrock D, et al. The Evolutionary History of 2,658 Cancers. Nature (2020) 578:122-8. doi: 10.1038/s41586-019-1907-7

35. Aptsiauri N, Cabrera T, Garcia-Lora A, Lopez-Nevot MA, Ruiz-Cabello F, Garrido F. MHC Class I Antigens and Immune Surveillance in Transformed Cells. Int Rev Cytol (2007) 256:139-89. doi: 10.1016/S0074-7696(07)56005-5

36. McGranahan N, Rosenthal R, Hiley CT, Rowan AJ, Watkins TBK, Wilson GA, et al. Allele-Specific HLA Loss and Immune Escape in Lung Cancer Evolution. Cell (2017) p. 1259-71. doi: 10.1016/j.cell.2017.10.001

37. Anichini A, Perotti VE, Sgambelluri F, Mortarini R. Immune Escape Mechanisms in Non Small Cell Lung Cancer. Cancers (Basel) (2020) 12. doi: $10.3390 /$ cancers 12123605

38. Tran E, Robbins PF, Lu Y-C, Prickett TD, Gartner JJ, Jia L, et al. T-Cell Transfer Therapy Targeting Mutant KRAS in Cancer. N Engl J Med (2016) 375:2255-62. doi: 10.1056/NEJMoa1609279

39. Hamburger AE, DiAndreth B, Cui J, Daris ME, Munguia ML, Deshmukh K, et al. Engineered T Cells Directed at Tumors With Defined Allelic Loss. Mol Immunol (2020) 128:298-310. doi: 10.1016/J.MOLIMM.2020.09.012

40. Hwang MS, Mog BJ, Douglass J, Pearlman AH, Hsiue EH-C, Paul S, et al. Targeting Loss of Heterozygosity for Cancer-Specific Immunotherapy. Proc Natl Acad Sci (2021) 118:e2022410118. doi: 10.1073/pnas.2022410118

41. Emami Nejad A, Najafgholian S, Rostami A, Sistani A, Shojaeifar S, Esparvarinha $M$, et al. The Role of Hypoxia in the Tumor Microenvironment and Development of Cancer Stem Cell: A Novel Approach to Developing Treatment. Cancer Cell Int (2021) 21:62. doi: 10.1186/s12935-020-01719-5

42. Tam SY, Wu VWC, Law HKW. Hypoxia-Induced Epithelial-Mesenchymal Transition in Cancers: HIF-1 $\alpha$ and Beyond. Front Oncol (2020) 10:486. doi: 10.3389/fonc.2020.00486

43. Wang B, Zhao Q, Zhang Y, Liu Z, Zheng Z, Liu S, et al. Targeting Hypoxia in the Tumor Microenvironment: A Potential Strategy to Improve Cancer Immunotherapy. J Exp Clin Cancer Res (2021) 40:1-16. doi: 10.1186/ s13046-020-01820-7

44. Grivennikov SI, Greten FR, Karin M. Immunity, Inflammation, and Cancer. Cell (2010) 140:883-99. doi: 10.1016/j.cell.2010.01.025

45. Landskron G, de la Fuente M, Thuwajit P, Thuwajit C, Hermoso MA. Chronic Inflammation and Cytokines in the Tumor Microenvironment. J Immunol Res (2014) 2014:1-19. doi: 10.1155/2014/149185

46. Lan T, Chen L, Wei X. Inflammatory Cytokines in Cancer: Comprehensive Understanding and Clinical Progress in Gene Therapy. Cells (2021) 10. doi: $10.3390 /$ cells 10010100

47. van Kempen LCL, de Visser KE, Coussens LM. Inflammation, Proteases and Cancer. Eur J Cancer (2006) 42:728-34. doi: 10.1016/j.ejca.2006.01.004

48. Mason SD, Joyce JA. Proteolytic Networks in Cancer. Trends Cell Biol (2011) 21:228-37. doi: 10.1016/j.tcb.2010.12.002

49. Sevenich L, Joyce JA. Pericellular Proteolysis in Cancer. Genes Dev (2014) 28:2331-47. doi: 10.1101/gad.250647.114

50. Nissim L, Bar-Ziv RH. A Tunable Dual-Promoter Integrator for Targeting of Cancer Cells. Mol Syst Biol (2010) 6:444. doi: 10.1038/msb.2010.99

51. Nissim L, Wu M-R, Pery E, Binder-Nissim A, Suzuki HI, Stupp D, et al. Synthetic RNA-Based Immunomodulatory Gene Circuits for Cancer Immunotherapy. Cell (2017) 171:1138-50.e15. doi: 10.1016/j.cell.2017.09.049

52. Xie M, Fussenegger M. Designing Cell Function: Assembly of Synthetic Gene Circuits for Cell Biology Applications. Nat Rev Mol Cell Biol (2018) 19:507-25. doi: 10.1038/s41580-018-0024-z

53. Wu M-R, Nissim L, Stupp D, Pery E, Binder-Nissim A, Weisinger K, et al. A High-Throughput Screening and Computation Platform for Identifying
Synthetic Promoters With Enhanced Cell-State Specificity (SPECS). Nat Commun (2019) 10:2880. doi: 10.1038/s41467-019-10912-8

54. Greenshpan Y, Sharabi O, Ottolenghi A, Cahana A, Kundu K, M. Yegodayev $\mathrm{K}$, et al. Synthetic Promoters to Induce Immune-Effectors Into the Tumor Microenvironment. Commun Biol (2021) 4:143. doi: 10.1038/s42003-02101664-7

55. Wilkie S, Van Schalkwyk MCI, Hobbs S, Davies DM, Van DS, Pereira ACP, et al. Dual Targeting of ErbB2 and MUC1 in Breast Cancer Using Chimeric Antigen Receptors Engineered to Provide Complementary Signaling. J Clin Immunol (2012) 32:1059-70. doi: 10.1007/s10875-012-9689-9

56. Kloss CC, Condomines M, Cartellieri M, Bachmann M, Sadelain M. Combinatorial Antigen Recognition With Balanced Signaling Promotes Selective Tumor Eradication by Engineered T Cells. Nat Biotechnol (2013) 31:71-5. doi: 10.1038/nbt.2459

57. Lanitis E, Poussin M, Klattenhoff AW, Song D, Sandaltzopoulos R, June CH, et al. Chimeric Antigen Receptor T Cells With Dissociated Signaling Domains Exhibit Focused Anti-Tumor Activity With Reduced Potential for Toxicity. Cancer Immunol Res (2013) 1:10. doi: 10.1158/2326-6066.CIR-13-0008

58. Globerson Levin A, Rawet Slobodkin M, Waks T, Horn G, Ninio-Many L, Deshet Unger N, et al. Treatment of Multiple Myeloma Using Chimeric Antigen Receptor T Cells With Dual Specificity. Cancer Immunol Res (2020) 8 (12):1485-95. doi: 10.1158/2326-6066.CIR-20-0118

59. Cho JH, Collins JJ, Wong WW. Universal Chimeric Antigen Receptors for Multiplexed and Logical Control of T Cell Responses. Cell (2018) 173 (6):1426-38. doi: 10.1016/j.cell.2018.03.038

60. Feldmann A, Hoffmann A, Bergmann R, Koristka S, Berndt N, Arndt C, et al. Versatile Chimeric Antigen Receptor Platform for Controllable and Combinatorial T Cell Therapy. Oncoimmunology (2020) 9:1785608. doi: 10.1080/2162402X.2020.1785608

61. Salzer B, Schueller CM, Zajc CU, Peters T, Schoeber MA, Kovacic B, et al. Engineering AvidCARs for Combinatorial Antigen Recognition and Reversible Control of CAR Function. Nat Commun (2020) 11:4166. doi: 10.1038/s41467-020-17970-3

62. Lajoie MJ, Boyken SE, Salter AI, Bruffey J, Rajan A, Langan RA, et al. Designed Protein Logic to Target Cells With Precise Combinations of Surface Antigens. Science (2020) 369:1637-43. doi: 10.1126/science.aba6527

63. Roybal KT, Rupp LJ, Morsut L, Walker WJ, McNally KA, Park JS, et al. Precision Tumor Recognition by T Cells With Combinatorial AntigenSensing Circuits. Cell (2016) 164:770-9. doi: 10.1016/j.cell.2016.01.011

64. Morsut L, Roybal KT, Xiong X, Gordley RM, Coyle SM, Thomson M, et al. Engineering Customized Cell Sensing and Response Behaviors Using Synthetic Notch Receptors. Cell (2016) 164:780-91. doi: 10.1016/ j.cell.2016.01.012

65. Roybal KT, Williams JZ, Morsut L, Rupp LJ, Kolinko I, Choe JH, et al. Engineering T Cells With Customized Therapeutic Response Programs Using Synthetic Notch Receptors. Cell (2016) 167:419-32.e16. doi: 10.1016/ j.cell.2016.09.011

66. Williams JZ, Allen GM, Shah D, Sterin IS, Kim KH, Garcia VP, et al. Precise T Cell Recognition Programs Designed by Transcriptionally Linking Multiple Receptors. Science (2020) 370:1099-104. doi: 10.1126/science.abc6270

67. Choe JH, Williams JZ, Lim WA. Engineering T Cells to Treat Cancer: The Convergence of Immuno-Oncology and Synthetic Biology. Annu Rev Cancer Biol (2020) 4:121-39. doi: 10.1146/annurev-cancerbio-030419-033657

68. Lal M, Caplan M. Regulated Intramembrane Proteolysis: Signaling Pathways and Biological Functions. Physiology (2011) 26:34-44. doi: 10.1152/ physiol.00028.2010

69. Hernandez-Lopez RA, Yu W, Cabral KA, Creasey OA, Lopez Pazmino MDP, Tonai $\mathrm{Y}$, et al. T Cell Circuits That Sense Antigen Density With an Ultrasensitive Threshold. Science (2021) 371:1166-71. doi: 10.1126/ science.abc1855

70. Juillerat A, Marechal A, Filhol JM, Valogne Y, Valton J, Duclert A, et al. An Oxygen Sensitive Self-Decision Making Engineered CAR T-Cell. Sci Rep (2017) 7:39833. doi: 10.1038/srep39833

71. Kosti P, Opzoomer JW, Larios-Martinez KI, Henley-Smith R, Scudamore CL, Okesola M, et al. Hypoxia-Sensing CAR T Cells Provide Safety and Efficacy in Treating Solid Tumors. Cell Rep Med (2021) 2:100227. doi: 10.1016/ j.xcrm.2021.100227 
72. Greenberger S, Shaish A, Varda-Bloom N, Levanon K, Breitbart E, Goldberg I, et al. Transcription-Controlled Gene Therapy Against Tumor Angiogenesis. J Clin Invest (2004) 113:1017-24. doi: 10.1172/JCI20007

73. Gruslova A, Cavazos DA, Miller JR, Breitbart E, Cohen YC, Bangio L, et al. VB-111: A Novel Anti-Vascular Therapeutic for Glioblastoma Multiforme. J Neurooncol (2015) 124:365-72. doi: 10.1007/s11060-015-1853-7

74. Autio KA, Boni V, Humphrey RW, Naing A. Probody Therapeutics: An Emerging Class of Therapies Designed to Enhance On-Target Effects With Reduced Off-Tumor Toxicity for Use in Immuno-Oncology. Clin Cancer Res (2020) 26:984-9. doi: 10.1158/1078-0432.CCR-19-1457

75. Desnoyers LR, Vasiljeva O, Richardson JH, Yang A, Menendez EEM, Liang TW, et al. Tumor-Specific Activation of an EGFR-Targeting Probody Enhances Therapeutic Index. Sci Transl Med (2013) 5:207ral44. doi: $10.1126 /$ scitranslmed.3006682

76. Han X, Bryson PD, Zhao Y, Cinay GE, Li S, Guo Y, et al. Masked Chimeric Antigen Receptor for Tumor-Specific Activation. Mol Ther (2017) 25:274-84. doi: 10.1016/j.ymthe.2016.10.011

77. Fedorov VD, Themeli M, Sadelain M. PD-1- and CTLA-4-Based Inhibitory Chimeric Antigen Receptors (iCARs) Divert Off-Target Immunotherapy Responses. Sci Transl Med (2013) 5:215ra172. doi: 10.1126/scitranslmed. 3006597

78. Tao L, Farooq MA, Gao Y, Zhang L, Niu C, Ajmal I, et al. CD19-CAR-T Cells Bearing a KIR/PD-1-Based Inhibitory CAR Eradicate CD19+HLA-C1-
Malignant B Cells While Sparing CD19+HLA-C1+ Healthy B Cells. Cancers (Basel) (2020) 12. doi: 10.3390/cancers 12092612

79. Cho JH, Okuma A, Sofjan K, Lee S, Collins JJ, Wong WW. Engineering Advanced Logic and Distributed Computing in Human CAR Immune Cells. Nat Commun (2021) 12:792. doi: 10.1038/s41467-021-21078-7

Conflict of Interest: The authors declare that the research was conducted in the absence of any commercial or financial relationships that could be construed as a potential conflict of interest.

Publisher's Note: All claims expressed in this article are solely those of the authors and do not necessarily represent those of their affiliated organizations, or those of the publisher, the editors and the reviewers. Any product that may be evaluated in this article, or claim that may be made by its manufacturer, is not guaranteed or endorsed by the publisher.

Copyright (c) 2021 Savanur, Weinstein-Marom and Gross. This is an open-access article distributed under the terms of the Creative Commons Attribution License (CC BY). The use, distribution or reproduction in other forums is permitted, provided the original author(s) and the copyright owner(s) are credited and that the original publication in this journal is cited, in accordance with accepted academic practice. No use, distribution or reproduction is permitted which does not comply with these terms. 